\title{
A Novel ACO with Average Entropy
}

\section{Yancang LI}

College of Civil Engineering, Hebei University of Engineering, Handan, China.

Email: liyancang@163.com

Received August $7^{\text {th }}, 2009$; revised September $1^{\text {st }}, 2009$; accepted September $14^{\text {th }}, 2009$.

\begin{abstract}
In order to solve the premature convergence problem of the basic Ant Colony Optimization algorithm, a promising modification with changing index was proposed. The main idea of the modification is to measure the uncertainty of the path selection and evolution by using the average information entropy self-adaptively. Simulation study and performance comparison on Traveling Salesman Problem show that the improved algorithm can converge at the global optimum with a high probability. The work provides a new approach for solving the combinatorial optimization problems, especially the NP-hard combinatorial optimization problems.
\end{abstract}

Keywords: ACO, Modification, Average Entropy, TSP

\section{Introduction}

Ant System (AS) algorithm proposed by Italy scholars Dorigo, Mahiezzo and Colorni in 1991 [1,2] is a new novel population-based meta-heuristic for solving the NP-hard combinatorial optimization problems. It belongs to the Ant Colony Optimization (ACO) which is a group of different ant-based approaches with different transition and pheromone updating rules. They combine distributed computation, autocatalysis (positive feedback) and constructive greedy heuristic in finding optimal solutions, and they are promising methods for solving the combinatorial optimization problems.

ACO has been successfully applied to the most combinatorial optimization problems, e.g. TSP (Traveling Salesman Problem) [3], JSP (Job-shop Scheduling Problem) [4], QAP (Quadratic Assignment Problem) [5,6] and so on [7-10]. Yet, because the ACO is still very young, it has many shortcomings, especially its premature convergence.

To break through this limitation, an improved ant colony algorithm based on the average information entropy is proposed here. The information entropy is used to judge the stability of the subspace of solutions represented at the given stage of algorithm's evolution and then it is applied to control the parameter of the algorithm. In some degrees, this work can solve the premature convergence of the basic ACO.

The rest of the paper is organized as follows. In Section 2, the theory and models of the basic ACO algorithm were introduced. In Section 3, attention was paid to the modification of the algorithm based on average entropy after brief introduction of the entropy. In the following part, simulation study and performance comparison with other ACO algorithms on the TSP were done and the direction of future research was pointed out.

\section{General Knowledge of Basic ACO}

As the other stimulated evolutionary algorithms, ACO is a family of meta-heuristics stochastic explorative algorithms inspired by real ants. It finds the best solution of optimization problem using the evolutionary procedure. As shown in [11], ACO is based on the following ideas. 1) From a starting point to an ending point, each path is associated with a candidate solution to a given problem. 2) The amount of pheromone deposited on each edge of the path followed by one ant is proportional to the quality of the corresponding candidate solution. 3) The edge with a larger amount of pheromone is chosen with higher probability. As a result, the ants eventually converge to a short path, hopefully the optimum or a near-optimum solution to the target problem.

The general framework of the ACO systems is:

Initialization

Repeat /*each iteration at this level is called acycle*/

Each ant is positioned on an arbitrary starting node

Repeat/*each iteration at this level is called a step*/

Each ant moves to next node according to the state transition rule

Until all ants have completed their tours

Apply the global pheromone updating rule Until end condition

As the basic ACO, Ant system (AS) uses the following 
analogies: artificial ants searching the solution space correspond to real ants searching their environment for food, the objective values are equivalent to the food sources quality and an adaptive memory represents the pheromone trails. The artificial ants are additionally equipped with a local heuristic function to guide their search through the set of feasible solutions. It was first applied to the several TSP problems of moderately smaller sizes (smaller than 75 cities).

The AS in Pseudo Code is:

\section{Initialize}

For $t=1$ to number of iterations do For $k=1$ to $m$ do

Repeat until ant $k$ has completed a tour

Select the city $j$ to be visited next with probability given

$$
\text { by equation of } p_{i j}^{k}=\left\{\begin{array}{l}
\frac{\tau_{i j}^{\alpha} \eta_{i j}^{\beta}(t)}{\sum_{s \in w_{k}} \tau_{i s}^{\alpha} \eta_{i s}^{\beta}(t)}, j \in w_{k} \\
0, \text { otherwise }
\end{array}\right.
$$

Calculate the length of tour generated by ant $k$ Update the trail levels on all edges according to equation

$$
\begin{gathered}
\tau_{i j}(t+n)=\rho \tau_{i j}(t)+\Delta \tau_{j} \\
\text { End }
\end{gathered}
$$

Where $\tau_{i j}$ is the intensity of trail between cities $\mathrm{i}$ and $\mathrm{j}$, $\alpha$ and $\beta$ are two adjustable parameters that control the relative weight of trail intensity and desirability, $\eta_{i j}$ is the visibility of city $\mathrm{j}$ from city $\mathrm{i}$ and $\eta_{i j}=\frac{1}{d_{i j}}, w_{k}$ is set of cities that have not been visited yet, and $\rho \in[0,1] \quad$ is the evaporation factor, $\Delta \tau_{j}$ is the change of trail level on edge $(i, j)$ caused by ant $\mathrm{k}$. According to the different forms of $\Delta \tau_{j}, \mathrm{M}$. Dorigo proposed three variants of its implementation: ant-cycle system, antquantity system and ant-density system.

The framework of the basic ACO can be shown in Figure 1.

In [12], the model of basic ACO was introduced. Herein they will not be repeated.

The ACO has been successfully applied to the most combinatorial optimization problems. Yet it has many shortcomings, especially its premature convergence.

With the attention and efforts of researchers in corresponding fields, AS algorithm is improved and expanded based on the initial model, such as the Ant System with elitist strategy (AS elite), Rank-Based Ant Systems (AS rank), Ant Colony System (ACS), MAX-MIN Ant System (MMAS) [13], Best-Worst Ant System, Graph-based Ant System, the candidate set strategies ACO, Ant-Q system [14], HC-ACO and so on. All of these were summarized in [12]. However, we still have a long way to process.

\section{Modification of the Basic ACO}

\subsection{Analysis of the Algorithm}

There are four main parts in basic ACO: selection strategy, local pheromone updating, local searching algorithm, and global pheromone updating. Herein, the positive feedback theory in the selection strategy is to reinforce the better solution, but it causes the stagnation behavior. This is the key to its shortage. In ACO, $\alpha$ and $\beta$ are two important adjustable parameters that control the relative weight of trail intensity and desirability. If $\alpha$ is big, the ant will seek for other routes. If $\beta$ is big, the ant will follow the shortest route found now, which will cause the premature. Usually, $\alpha$ and $\beta$ are constant, which influence the performance of the algorithm. Here, an algorithm with changing index will be proposed.

The average information entropy was introduced to measure the uncertainty of the selection. As a physical concept, entropy was first proposed by Clausius to describe the confusion or "disorder" of the system [15]. Then it was introduced into many fields and many concepts of entropy were presented, e.g. L. Boltzman Entropy, Information Entropy, Probability Entropy, and Topological Entropy and so on. In 1948, Shannon defined the concept of information entropy to weigh the uncertainty of the information and selection. It is an important concept and has been widely used to weigh the uncertainty of the system.

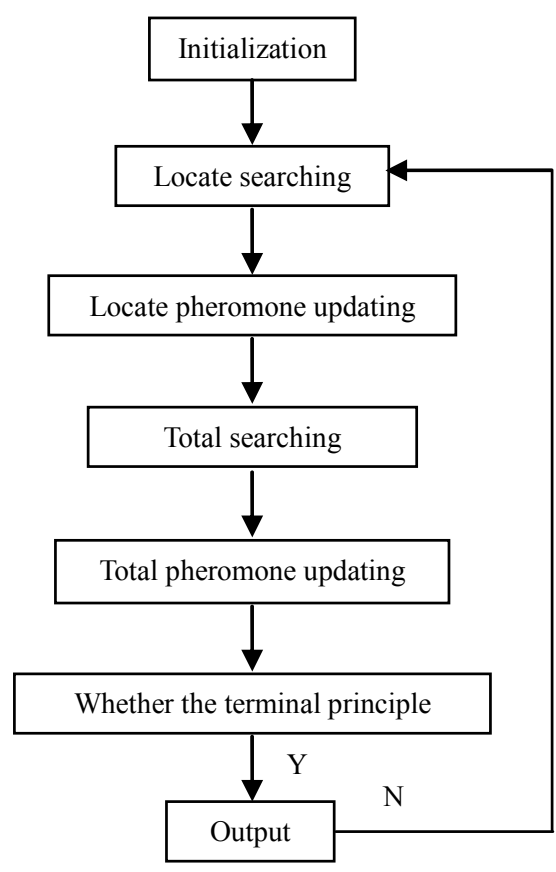

Figure 1. Framwork of basic ACO 
For the discrete stochastic variables, the information entropy is:

$$
S=-k \sum_{i=1}^{n} p_{i} \ln p_{i}
$$

Where $p_{i}$ is the probability of the state, and $p_{i} \geq 0$, $\sum_{i=1}^{n} p_{i}=1$. And $k$ is a constant weight, and $k \succ 0$.

The information entropy has the following characteristics:

1) Symmetrical characteristic: the value of the information does not rely on the order of $p_{i}$ :

$$
S\left(p_{1}, p_{2}, \cdots, p_{n}\right)=S\left(p_{n}, p_{n-1}, \cdots, p_{1}\right)
$$

2) Additive characteristic: the sum of the independent state's information entropy is equal to the total state's information entropy.

3) Extreme property characteristic. When $p_{i}=\frac{1}{n}$, the information entropy reaches its biggest value $\ln n$.

4) Nonnegative characteristic. $S\left(p_{1}, p_{2}, \cdots, p_{n}\right) \geq 0$.

The basic ACO has a vital limitation: premature convergence. The key to the shortage is the positive feedback theory in the selection strategy. So we can control $\alpha$ and $\beta$ to avoid the premature convergence.

Define the selection probability is $p_{i j}^{k}(t)$, using the formula of entropy, $S=-k \sum_{i=1}^{n} p_{i} \ln p_{i}$, and then every ant will have a entropy $S_{i(t)}$. And the average entropy $\bar{S}=\frac{S}{m}$ can be used to define the uncertainty of selection:

$$
\begin{gathered}
\alpha_{(t)}=A\left|\bar{S}-S_{(t)}\right| \\
\beta_{(t)}=B-\left(\bar{S}-S_{(t)}\right)
\end{gathered}
$$

Where $A$ and $B$ are constant weight, and simulation experiment shows that when $A=2, B=10$, the algorithm performs best.

Therefore, we can control the entropy to ensure that at the beginning of the algorithm, $\alpha_{(t)}$ is small, and at the last, $\alpha_{(t)}$ increases. At the same time, $\beta_{(t)}$ is biggest at early stage in order to make the algorithm find the optimal route and later it becomes smaller to reinforce the function of random operation. By controlling the value of information entropy, we can control the route selection and the proportion of the stochastic local, and avoid the perturbation behavior.
For the terminal principle, the general algorithms usually use the maximum iteration time. Herein we employ the information entropy as the termination constraint because, in some cases, it is very difficult to decide the maximum iterative time for the complicated problems. We define when the information entropy is smaller than a given value (such as 0.01 ), the algorithm terminates and the solution is obtained.

\subsection{Frame of the Improved Algorithm Proposed Here}

The procedure of the modification we proposed is shown in Figure 2.

\section{Performance Comparison}

To validate the efficiency of the modification proposed, we compared its performance with other ACO algorithms and various heuristic and meta-heuristic search techniques including similar methods in other strategies. All algorithms were benchmarked on TSP of variable sizes with known optimal solutions.

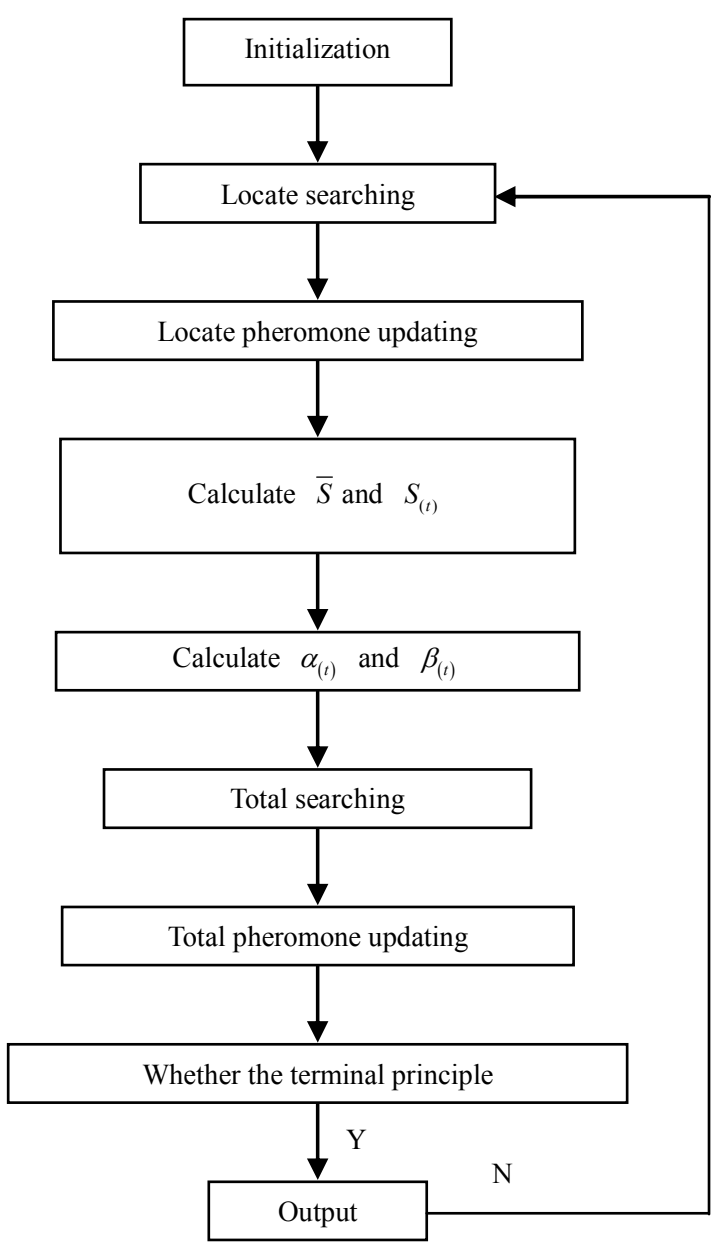

Figure 2. The procedure of the modification 
Table 1. The results of different algorithms

\begin{tabular}{rccccc}
\hline & optimum & Algorithm proposed here & Ant System & Ant Colony Algorithm & Rank Ant System \\
\hline TSP-50 & 425 & 424.7 & 427.3 & 425.3 & 425.9 \\
TSP-198 & 15780 & 15807.2 & 16702.1 & 16540.4 & 15960.4 \\
TSP-442 & 50778 & 51002.8 & 54310.3 & 51691.2 & 51228.5 \\
TSP-657 & 48912 & 49309.8 & 52124.7 & 51197.3 & 49382.1 \\
TSP-1577 & 22249 & 22257.3 & 26063.7 & 23163.2 & 22340.8 \\
\hline
\end{tabular}

The choice of TSP for the performance comparison is explained as: 1) this is the first combinatorial problem that was solved by ant algorithms; 2) this is a traditional benchmark problem for combinatorial optimization methods. There is a big library of test traveling sales-man problems and methods of their solution, which makes it possible to compare the efficiency of the ant algorithms with other optimization approaches; 3) the NP-hard problem can conveniently be interpreted in terms of the ant behavior.

Herein, terminal constraint of the algorithm proposed here is $\mathrm{H}(\mathrm{t})<0.01$, the reason for these choices were because previous tests indicated they were enough to achieve good solutions on the questions used. Table 1 summarizes the results of the study.

Using the method above, we finished the safety assessment of a jacket installation procedure of an offshore oil field in South China Sea. The installation procedure contains the lifting installation, jacket launch and upending operations. The scores of every factor given by the specialists are listed in Table 1.

As shown in Table 1, AS performed well for small problems(less than 75 cities), but its performance was not good for larger problems. Rank Ant System was the second best. Ant Colony Algorithm performed better than all algorithms. The algorithm proposed here could find results which are at least as good as, and often better than those found by the other ACO algorithms, especially for the big problem (larger than 442). It was the best performer. This is mainly contributed to the good exploration and intensification by using the parameters using the information average entropy.

\section{Conclusions}

One of the most common problems encountered by the ACO algorithms is the premature deficiency. The main contribution of this paper is a study of the avoidance of stagnation behavior by using the average entropy. The simulation study and performance comparison with other algorithms showed that the algorithm proposed here is a good and promising idea. The work gives rise to the

\section{ACO algorithms.}

The study of ACO is in constant development, and there is still a wealth of discoveries to be made.

Simulation study demonstrates that different values of parameters in one algorithm provides different results, more experiments need to be done in this area and more complete evidence about the comparative results should be given in the future.

\section{REFERENCES}

[1] M. Dorigo, V. Maniezzo, and A. Colorni, "The ant system: An autocatalytic optimizing process," Technical Report 91-106 revised, Department of Electronic, Politecnico of Milano, Milan, Italy, 1991.

[2] Colorni, M. Dorigo, and V. Maniezzo, "Distributed Optimization by Ant Colonies," in Proceedings of the First European Conference on Artificial Life, Elsevier Publishing, Paris, France, 1991.

[3] M. Dorigo and L. M. Gambardella, "Ant colony system: A cooperative learning approach to the traveling salesman problem," IEEC Transon Evolutionary Computing, Vol. 1 No. 1, pp. 53-56, 1997.

[4] Blum, A. Roli and M. Dorigo, "HC-ACO: The hyper-cube framework for ant colony optimization," in Proceedings of MIC 2001-meta-heuristics International Conference, Porto, Portugal, Vol. 2, pp. 399-403, 2001.

[5] A. Colorni, M. Dorigo, and V. Maniezzo, "Ant colony system for job-shop scheduling," Belgian Journal of Operations Research Statistics and Computer Science, Vol. 34, No. 1, pp. 39-53, 1994.

[6] V. Maniezzo, M. Dorigo, and A. Colorni, "The ant system applied to quadratic assignment problem," Technical Report IRIDIA94-28, University de Bruxelles, Belgium, 1994.

[7] L. Gambardella and M. Dorigo, "HAS-SOP: Hybrid ant system for the sequential problem," Technical Report, IDSIA, 1997.

[8] L. Chen and Z. Pan, "Ant colony optimization approach for test scheduling of system on chip," Journal of Chongqing University of Posts and Telecommunications, Vol. 21, No. 2, pp. 212-217, 2009.

[9] M. L. Spangler, K. R. Robbins, J. K. Bertrand, and M. Macneil, et al., "Ant colony optimization as a method for 
strategic genotype sampling," Animal genetics, Vol. 40, No. 3, pp. 308-314, 2009.

[10] Q. Zhang, "Research on ant colony algorithm and its applications", Computer Knowledge and Technology, Vol. 5, No. 9, pp. 2396-2398, 2009.

[11] M. Dorigo and T. Stutzle, "Ant colony optimization," Cambridge, MIT Press, MA, 2004.

[12] Y. Li and W. Li, "Adaptive ant colony optimization algorithm based on information entropy: Foundation and application," Fundamenta Informaticae, Vol. 77, No. 3, pp. 229-242, 2007
[13] T. Stutzle and H. Hoos, "MAX-MIN ant system," Future Generation Computer systems, No. 16, pp. 889-914, 2000.

[14] M. Dorigo and M. Luca, "A study of some properties of Ant-Q," Proceedings of 4th International Conference on Parallel Problem from Nature, Berlin: Springer Verlag, pp. 656-665, 1996.

[15] Wanhua Q., "Management decision making and the applied entropy," China Mechanical Press, Beijing, China, 2002. 